\title{
Computer Simulation of Backscattering Spectra from Paint
}

\author{
M. Mayer ${ }^{\mathrm{a}}$, T.F. Silva ${ }^{\mathrm{a}, \mathrm{b}}$ \\ ${ }^{a}$ Max-Planck-Institut für Plasmaphysik, Boltzmannstr. 2, 85748 Garching, Germany \\ ${ }^{b}$ Instituto de Física da Universidade de São Paulo, Rua do Matão, trav. R 187, 05508-090 São \\ Paulo, Brazil
}

\begin{abstract}
To study the role of lateral non-homogeneity on backscattering analysis of paintings, a simplified model of paint consisting of randomly distributed spherical pigment particles embedded in oil/binder has been developed. Backscattering spectra for lead white pigment particles in linseed oil have been calculated for $3 \mathrm{MeV} \mathrm{H}^{+}$at a scattering angle of $165^{\circ}$ for pigment volume concentrations ranging from $30 \mathrm{vol} \%$ to $70 \mathrm{vol} . \%$ using the program STRUCTNRA. For identical pigment volume concentrations the heights and shapes of the backscattering spectra depend on the diameter of the pigment particles: This is a structural ambiguity for identical mean atomic concentrations but different lateral arrangement of materials. Only for very small pigment particles the resulting spectra are close to spectra calculated supposing atomic mixing and assuming identical concentrations of all elements.

Generally, a good fit can be achieved when evaluating spectra from structured materials assuming atomic mixing of all elements and laterally homogeneous depth distributions. However, the derived depth profiles are inaccurate by a factor of up to 3 . The depth range affected by this structural ambiguity ranges from the surface to a depth of roughly 0.5 to 1 pigment particle diameters. Accurate quantitative evaluation of backscattering spectra from paintings therefore requires taking the correct microstructure of the paint layer into account.
\end{abstract}

Keywords: Ion beam analysis; Lateral inhomogeneity; STRUCTNRA; Paint 


\section{Introduction}

Ion beam analysis (IBA) methods are often applied for the quantitative elemental analysis of objects from cultural heritage, such as archaeological artefacts or paintings [1,2]. Typically an external beam of 2-3 MeV protons is used [3] and elastic backscattering spectroscopy (EBS), particle induced X-ray emission (PIXE) and particle induced $\Gamma$-ray emission (PIGE) data are combined [4]. The measured spectra are being used for quantitative depth profiling of elements and for the determination of total amounts of trace elements [5]. Different beam types (for example ${ }^{4} \mathrm{He}$ beams) are sometimes used alternatively or complementary. The EBS spectra provide quantitative information about concentration and depth profiles of light elements (especially $\mathrm{C}$ and $\mathrm{O}$ ) and of the main heavy constituents, while the PIXE/PIGE spectra are typically used to identify trace elements.

On paintings, the paint layer typically consists of color pigments in the form of powder embedded in oil or binder. Linseed oil, a mixture of 52-55\% linolenic acid $\mathrm{C}_{18} \mathrm{H}_{30} \mathrm{O}_{2}, 18-23 \%$ oleic acid $\mathrm{C}_{18} \mathrm{H}_{34} \mathrm{O}_{2}$ and $14-17 \%$ linoleic acid $\mathrm{C}_{18} \mathrm{H}_{32} \mathrm{O}_{2}$, is often used, but historically other components (for example egg) have been also employed. Many different types of pigments have been used during the last few hundred years [6]. Some pigments are low- or medium-Z materials such as charcoal or titanium white $\mathrm{TiO}_{2}$, but most color pigments contain high-Z elements such as lead white $\left(\mathrm{PbCO}_{3}\right)_{2} \bullet \mathrm{Pb}(\mathrm{OH})_{2}$ or $\mathrm{PbSO}_{4} \bullet 3 \mathrm{PbO} \cdot \mathrm{H}_{2} \mathrm{O}$, lithophone white $\mathrm{BaSO}_{4}$ $+\mathrm{ZnS}$, cadmium orange/red $\mathrm{CdS}+\mathrm{CdSe}$ and many others. The diameter of the pigment particles can vary over a wide range from several $100 \mathrm{~nm}$ to several $10 \mu \mathrm{m}[7,8]$, the volume fraction of the pigments (usually called pigment volume concentration (PVC)) is typically between 20 and 70 vol.\% [7, 9].

This microstructure of paint is usually neglected in the analysis of IBA spectra. Popular simulation codes for the evaluation of IBA spectra [10] assume atomic mixing of all elements. The distribution of elements is assumed to be homogeneous in lateral direction (i.e. parallel to the surface) and to vary only with depth (i.e. perpendicular to the surface): The distribution of elements then can be described by concentration depth profiles for each element.

However, as has been already demonstrated in [11] the analysis of backscattering spectra from heterogeneous multi-phase materials such as paint can get ambiguous and extracted depth profiles can get inaccurate if a multi-phase material with lateral distribution of materials is approximated to be laterally homogeneous: This approximation is only valid if the constituents 
are sufficiently small but fails if the constituents get larger. This is a structural ambiguity and represents a potentially serious problem for the quantitative application of IBA methods to cultural heritage objects like paintings.

The examples in [11] are, however, idealized and not directly applicable to paint layers. This paper therefore presents a more realistic model for the microstructure of paint and investigates the consequences for the quantitative analysis of EBS spectra from lead white pigment particles in linseed oil.

\section{Computer simulation}

\subsection{Simulation of spectra}

EBS spectra from idealized paint layers were calculated using the program STRUCTNRA [11]. SIMNRA 6.98 [12, 13] was used as simulation kernel. SRIM-2003 stopping powers [14] and SigmaCalc non-Rutherford scattering cross-sections from carbon and oxygen were used [15]. Multiple small-angle scattering was taken into account as energy spread, dual large-angle scattering [16] was neglected. An intercomparison between the codes STRUCTNRA [11], RBS-MAST [17], CORTEO [18] and F95-Rough [19] showed very good agreement between all codes as well as with experimental data of ${ }^{4} \mathrm{He}$ ions backscattered from a grid structure [20]. The sample structure was created as described in section 2.2 and was imported into STRUCTNRA in 24-bit bitmap (BMP) format. Each color in the image represented a material consisting of its elements and a mass density. Pixels were assumed to be squares, periodic boundary conditions were applied in horizontal direction. Trajectories of incident particles started at quasirandom starting points at the upper edge of the image aiming towards the bottom edge.

Depth profiles were derived from the spectra using MultiSIMNRA [21] assuming atomic mixing of all elements. The same input parameters (stopping powers, cross-sections, particles times steradians etc.) as in the STRUCTNRA simulations were used.

\subsection{Microstructure of paint}

An idealized model of paint has been implemented. Paint is represented as a three-dimensional random distribution of spherical equally sized color pigment particles embedded in a matrix of oil/binder, see Fig. 1. The sample volume has an adjustable number of pixels in horizontal and vertical directions; the perpendicular direction (i.e. perpendicular to the paper plane in Fig. 1) 
has a size of two pigment particle diameters. Periodic boundary conditions are applied in horizontal and perpendicular direction. Only the central plane of the sample volume is used for further simulations by STRUCTNRA and is shown in Fig. 1. Due to the random arrangement of the spheres in 3 dimensions the diameter of the circles visible in the cross-sectional plane shown in Fig. 1 varies depending on the perpendicular position of the sphere relative to the paper plane.

The arrangement of the spherical pigment particles can follow either overlapping or non-overlapping models, see Fig. 1. Within the overlapping model pigment volume fractions from $0-100$ vol.\% can be realized, the non-overlapping case allows only pigment volume fractions up to about 35 vol. $\%$.

Three different surface models are available representing sample surfaces generated by cutting, by submersion, or by stick-out, see Fig. 1. The first model allows pigment particles to be cut at the surface and is suitable for samples originating from a multi-phase solid-state material manufactured by paring processes like sawing, grinding, or polishing. The depth profile of the mean pigment concentration is constant until the surface. The second model leaves the pigment spheres intact and fully submersed below the surface. This usually represents a glossy paint finishing [22]. In this model the mean pigment concentration drops to zero at the surface. The third surface model is a stick-out model where pigment particles may stick out from the surface by some fraction of their diameter. This model represents semi-gloss and flat paint finishing [22].

\section{Results and discussion}

Typical sample structures for a pigment volume concentration of 30 vol.\% and $2.5 \mu \mathrm{m}, 10 \mu \mathrm{m}$ and $50 \mu \mathrm{m}$ pigment particle diameter are shown in Fig. 2 (left). The samples have overlapping pigment particles and a cut through the pigments at the surface. All samples consist of linseed oil with a mean composition of $\mathrm{C}_{18} \mathrm{H}_{31} \mathrm{O}_{2}$ and a mass density of $0.93 \mathrm{~g} / \mathrm{cm}^{3}$ (grey) and lead white $2 \mathrm{PbCO}_{3} \cdot \mathrm{Pb}(\mathrm{OH})_{2}$ with a mass density of $6.14 \mathrm{~g} / \mathrm{cm}^{3}$ (white), see Table 1. Sample sizes used for the simulations were 4096x100 pixels with a pixel size of $500 \mathrm{~nm}$, corresponding to sample sizes of $2048 \times 50 \mu \mathrm{m}^{2}$. Due to the size of the samples only about $10 \%$ of their horizontal extension is shown in Fig. 2.

Simulated spectra for $3 \mathrm{MeV}$ incident protons at a scattering angle of $165^{\circ}$ are shown in Fig. 2 (right) for the different samples. A detector resolution of $15 \mathrm{keV}$ was assumed. The spectrum 
for infinitesimally small pigment particles corresponds to atomic mixing of all elements and was calculated using SIMNRA, all other spectra were calculated using STRUCTNRA.

For $2.5 \mu \mathrm{m}$ pigment particle diameter the spectrum is close to the spectrum for infinitesimally small pigment particles, only the part of the spectrum close to the high energy edge shows some differences. For increasing pigment particle diameter the spectra show increasingly larger differences, especially for the $\mathrm{Pb}$ signal. It should be kept in mind that the mean sample composition does not change with changing pigment particle diameter but is constant throughout the whole sample depth at a pigment volume concentration of $30 \mathrm{vol} \%$ (corresponding to 23.1 at.\% lead white, see Table 1). Different spectra for identical mean sample composition but different lateral arrangement of elements represent a structural ambiguity as already described in [11] for a chessboard-like arrangement of elements.

Fig. 3 shows two simulated spectra for $3 \mathrm{MeV} \mathrm{H}^{+}$backscattered from lead white in linseed oil. Black hollow dots mark the spectrum for a pigment volume concentration of $30 \mathrm{vol} . \%$ and a pigment particle diameter of $25 \mu \mathrm{m}$. This spectrum is already shown in Fig. 2 (orange line). The black line in Fig. 3 is a spectrum calculated for atomic mixing of $\mathrm{H}, \mathrm{C}, \mathrm{O}$, and $\mathrm{Pb}$ using the concentration depth profile shown in Fig. 4 (orange line). Both spectra are practically indistinguishable. In the depth profile the atomic concentration of lead white decreases to about 10 at.\% at the surface while the true mean atomic concentration of lead white in this sample is constant 23.1 at.\% until the sample surface. This example shows that EBS spectra from heterogeneous materials like paint are generally ambiguous: The same spectrum can be obtained from a heterogeneous arrangement of the materials in different phases or from an atomic mix of the constituting elements with some depth distribution. But in general the mean atomic concentrations are not identical! Analysis of an EBS spectrum from a heterogeneous sample assuming atomic mixing of the constituting elements therefore in general will result in quantitatively incorrect results. This is the structural ambiguity already discussed in [11]. It is basically a stopping power effect. Identical problems therefore have to be expected also for NRA or ERD spectra.

Depth profiles derived from the spectra shown in Fig. 2 assuming atomic mixing are shown in Fig. 4. In the vicinity of the surface the derived atomic concentration of the pigment is always smaller than the real concentration, for pigment particle diameters of 25 and $50 \mu \mathrm{m}$ the deviation of the derived pigment concentration from the real concentration is within a factor of 2-3. It has to be stressed again that this deviation is observed despite the fact that the spectrum 
based on the depth profile and the spectrum based on spherical pigment particles are almost indistinguishable, as shown as example in Fig. 3. The depth range where an incorrect pigment concentration is derived ranges from the very surface to a depth of roughly one sphere diameter. In larger depths the correct pigment concentration is obtained.

Typical sample structures and simulated spectra for $3 \mathrm{MeV} \mathrm{H}^{+}$backscattered from lead white in linseed oil are shown in Fig. 5 for a pigment volume concentration of 50 vol.\%. The true mean atomic concentration of lead white in all samples is 41.2 at.\%, see Table 1. Depth profiles derived from the spectra shown in Fig. 5 assuming atomic mixing of all constituting elements are shown in Fig. 6. In the vicinity of the surface the derived atomic concentration of the pigment is always smaller than the real concentration, for pigment particle diameters of 25 and $50 \mu \mathrm{m}$ the deviation of the derived pigment concentration from the real concentration is within a factor of about 2 .

The same as above is shown in Figs. 7 and 8 for a pigment volume concentration of 70 vol.\%. The true mean atomic concentration of lead white in these samples is 62.1 at.\%, see Table 1 . In the vicinity of the surface the derived atomic concentration of the pigment is always smaller than the real concentration, for pigment particle diameters of 25 and $50 \mu \mathrm{m}$ the deviation of the derived pigment concentration from the real concentration is within a factor of about 1.5. Typical sample structures and simulated spectra for $3 \mathrm{MeV} \mathrm{H}^{+}$backscattered from lead white in linseed oil are shown in Fig. 9 for a pigment volume concentration of 30 vol.\%, non-overlapping pigment particles, and the submersion model with $5 \mu \mathrm{m}, 10 \mu \mathrm{m}$ and $25 \mu \mathrm{m}$ pigment particle diameter. The true mean atomic concentration of lead white is shown in Fig. 10 as dashed lines: Within the submersion model the lead white concentration drops to zero at the very surface and reaches a maximum at a depth of slightly less than one pigment particle diameter. At larger depths the pigment volume concentration reaches the mean value of 23.1 at.\%. Depth profiles derived from the spectra shown in Fig. 9 assuming atomic mixing of all constituting elements are shown in Fig. 10. In the vicinity of the surface the derived atomic concentration of the pigment is always smaller than the real concentration, for pigment particle diameters of $25 \mu \mathrm{m}$ the deviation of the derived pigment concentration from the real concentration is within a factor of about 2. As a consequence of the too low pigment concentration close to the surface the concentration maximum appears deeper in the sample by about $30 \%$. 
The examples shown in Figs. 2 to 10 demonstrate that this structural ambiguity exists for different models of the paint structure and through a wide range of pigment volume concentrations from 30 to $70 \mathrm{vol} \%$. The latter pigment concentrations are already higher than the critical pigment volume concentration (CPVC), which is about 46 vol.\% for lead white. Assuming atomic mixing the derived lead white concentration close to the surface is always too low. For the investigated volume concentrations this effect is highest at $30 \mathrm{vol} . \%$, where the lead white concentration is underestimated by a factor of almost 3 . At 70 vol.\% the effect still exists but gets smaller and the underestimation is by a factor of about 1.5. The affected depth range is about 0.5 to 1 pigment particle diameters, at larger depths the correct lead white concentration is obtained.

\section{Conclusions}

A simplified model of paint assuming spherical pigment particles embedded in oil/binder has been developed. The pigment particles are randomly distributed in 3 dimensions and may either overlap or not. At the surface the pigment particles are either cut, fully submersed, or may stick out by some fraction of their diameter.

Backscattering spectra for lead white pigment particles in linseed oil have been calculated for $3 \mathrm{MeV} \mathrm{H}^{+}$at a scattering angle of $165^{\circ}$ for pigment volume concentrations from 30 vol.\% to 70 vol.\%. For identical mean pigment volume concentrations the heights and shapes of the backscattering spectra depend on the diameter of the pigment particles: This is a structural ambiguity for identical mean atomic concentrations but different lateral arrangement of the materials. Only for very small pigment particles the spectra are identical to spectra assuming atomic mixing of all elements.

If spectra are evaluated neglecting this microstructure and assuming atomic mixing of all elements, then generally a good fit can be achieved. However, despite the good fit the derived depth profiles can be inaccurate by a factor of up to 3 for the lead white concentration because identical spectra can be obtained for different mean atomic concentrations if the lateral arrangement of the elements is different. The depth range affected by this structural ambiguity ranges from the surface to a depth of 0.5 to 1 pigment particle diameters. Accurate quantitative evaluation of backscattering spectra from paint therefore requires taking the correct microstructure of paint into account. Neglecting this microstructure and assuming atomic mixing is only justified if the pigment particles are sufficiently small. However, even in this 
case the near surface layer within 0.5 to 1 pigment particle diameters may give incorrect atomic concentrations. For quantitatively accurate results it is therefore advisable to accompany IBA investigations of paint by optical or electron microscopy investigations of the microstructure of the material. 
Overlapping
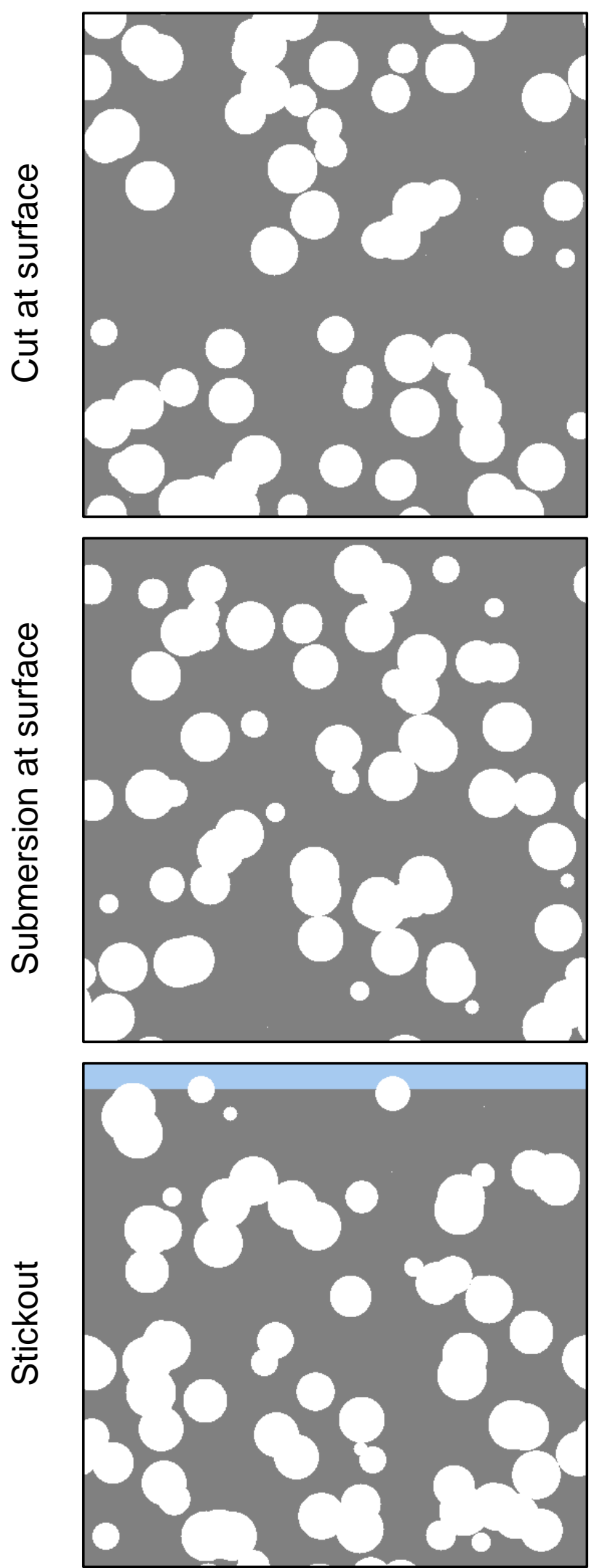

Non-Overlapping
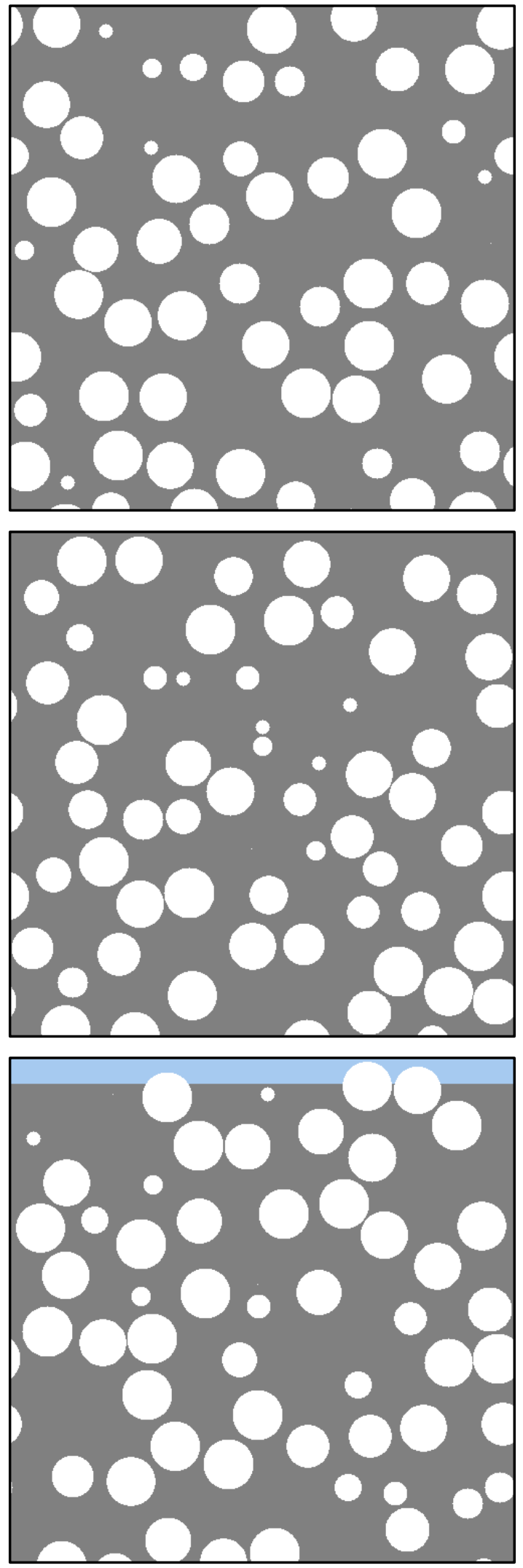
Fig. 1: Idealized models of paint consisting of randomly distributed spherical pigment particles (white) in a matrix of a different material (grey). Sky blue: Empty. The pigment particles are randomly distributed in 3 dimensions; the figure shows the central plane of the simulated volumetric samples. The surface is at the top. The pigment volume concentration is $30 \mathrm{vol} . \%$. The size of each image is 500x500 pixels; the pigment particle diameter is 50 pixels. Left column: Overlapping pigment particles; Right column: Non-overlapping pigment particles. Top row: Cut of pigment particles at the surface; Middle row: Submersion of pigment particles at the surface; Bottom row: Stick-out of pigment particles at the surface with a maximum stick-out of $50 \%$ of their diameter. 

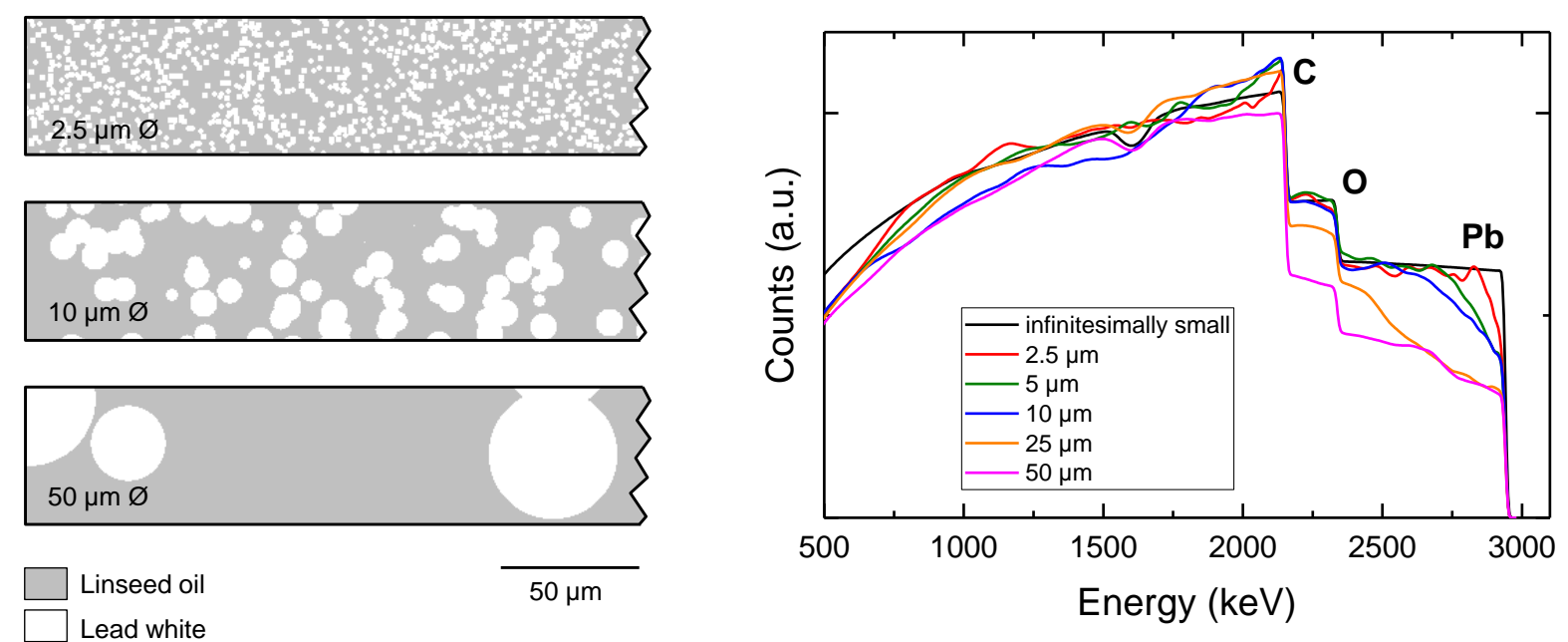

Fig. 2: Left: Typical sample structures for a pigment volume concentration of 30 vol.\% and $2.5 \mu \mathrm{m}, 10 \mu \mathrm{m}$ and $50 \mu \mathrm{m}$ pigment particle diameter. Grey: Linseed oil; White: Lead white. Sample sizes 4096x100 pixels with a pixel size of $500 \mathrm{~nm}$, corresponding to sample sizes of $2048 \times 50 \mu \mathrm{m}^{2}$. Only a small fraction of each sample is shown. Right: Simulated spectra for $3 \mathrm{MeV}$ incident protons at a scattering angle of $165^{\circ}$ for infinitesimally small pigment particles (i.e. atomic mixing of all elements) and pigment particle diameters from $2.5 \mu \mathrm{m}$ to $50 \mu \mathrm{m}$. The edges of $\mathrm{C}, \mathrm{O}$ and $\mathrm{Pb}$ are indicated. 


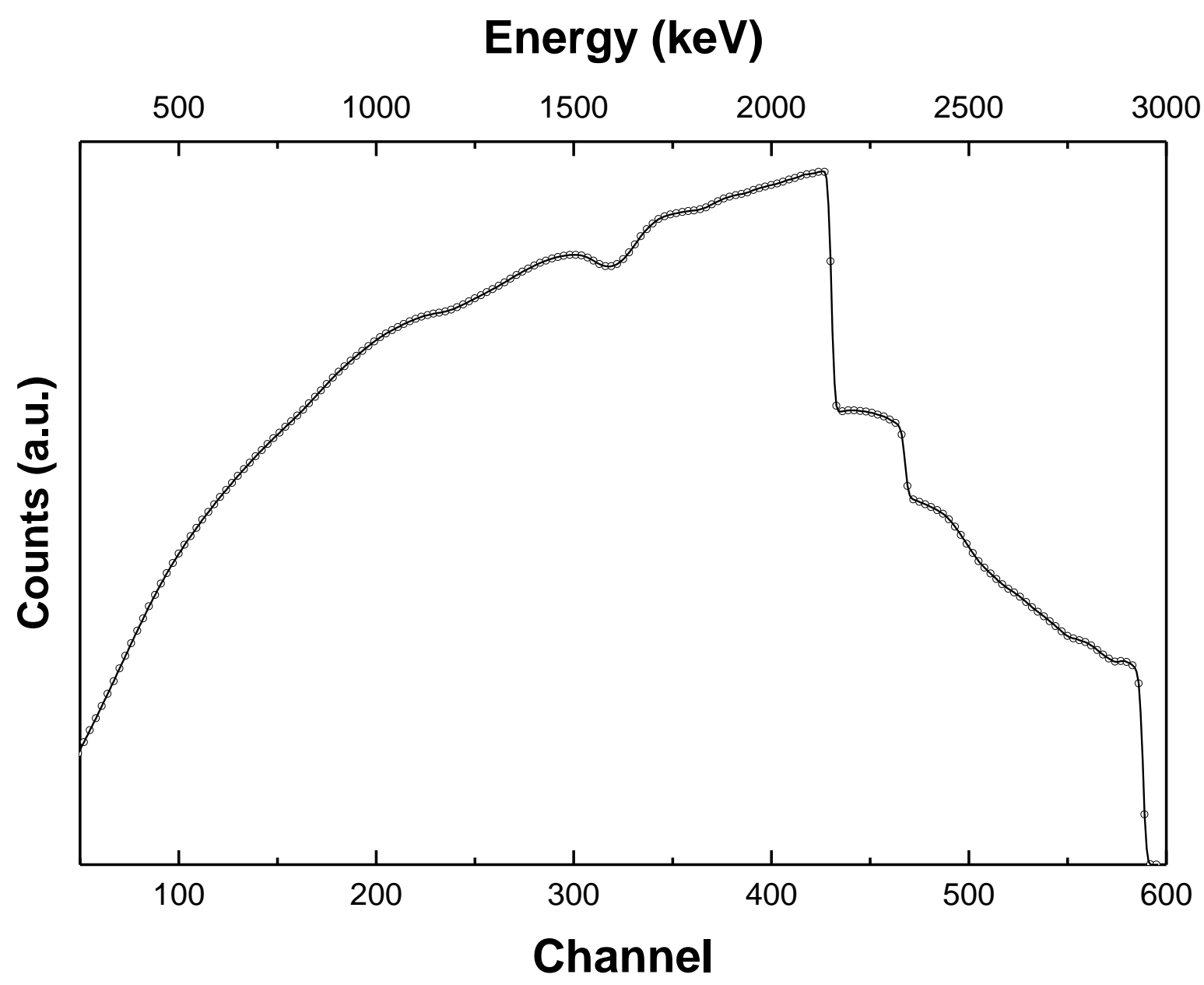

Fig. 3: Simulated spectra for $3 \mathrm{MeV} \mathrm{H}^{+}$backscattered from lead white in linseed oil, scattering angle $165^{\circ}$. Black hollow dots: Pigment volume concentration 30 vol.\%, pigment particle diameter $25 \mu \mathrm{m}$, overlap of pigment particles, cut at surface (identical to the orange line in Fig. 2). For better visibility only every third point is shown. Black line: Atomic mixing of $\mathrm{H}, \mathrm{C}$, $\mathrm{O}$ and $\mathrm{Pb}$ according to the depth profile shown in Fig. 4 (orange line). 
Depth $(\mu \mathrm{m})$

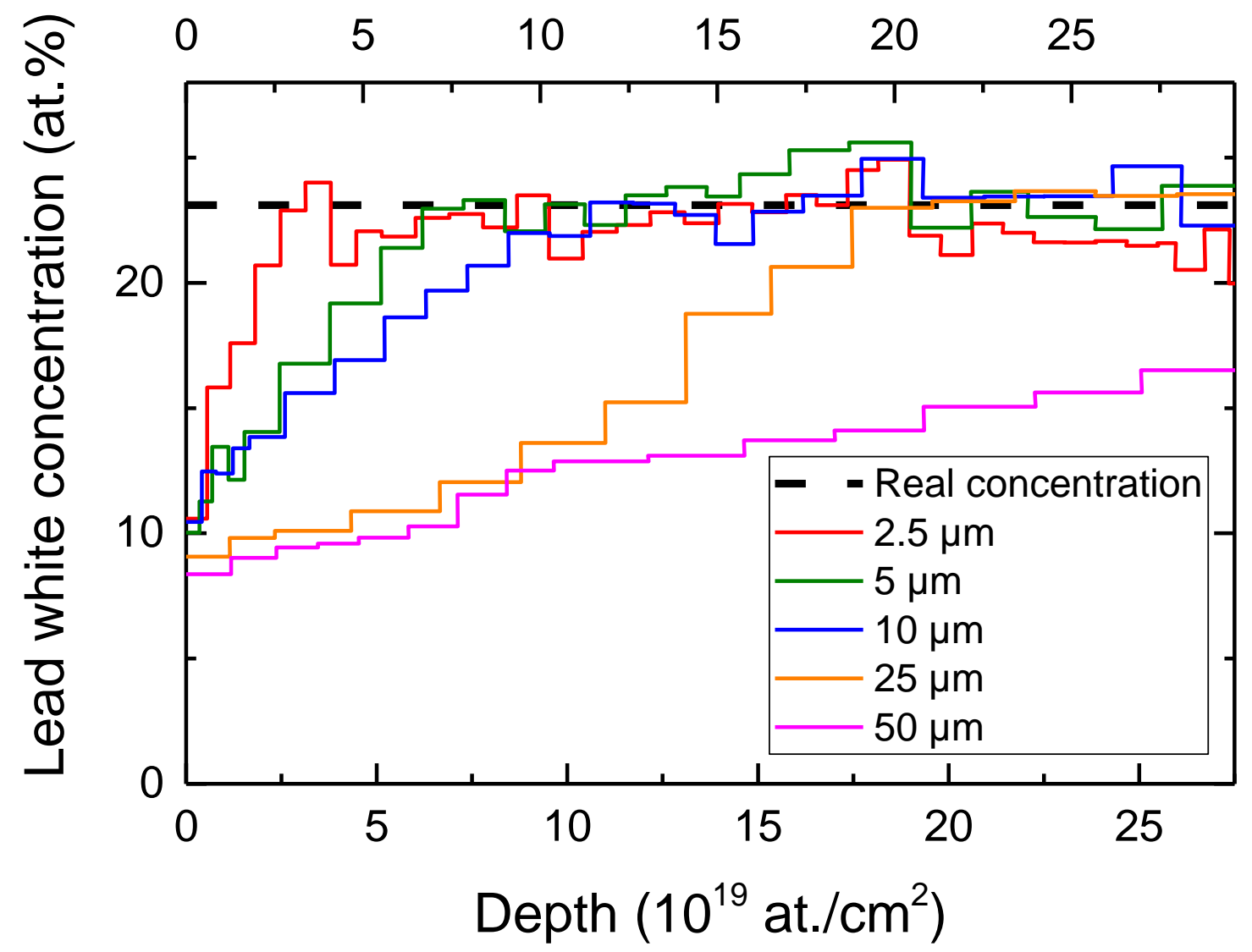

Fig. 4: Depth profiles of lead white in linseed oil derived by fitting a depth profile to the spectra shown in Fig. 2 (see Fig. 3 for the $25 \mu \mathrm{m}$ diameter case). Dashed line: Real atomic concentration of lead white; Solid lines: Lead white atomic concentrations derived from the spectra for different pigment particle diameters. 

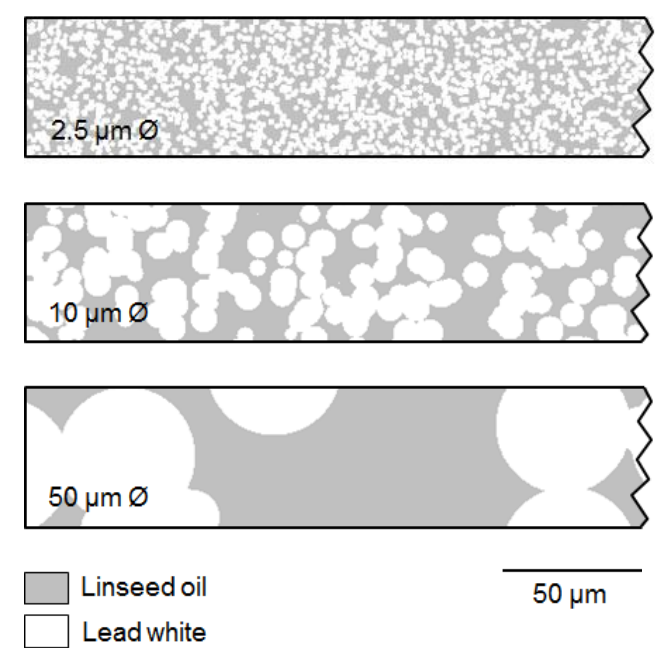

Fig. 5: Left: Typical sample structures for a pigment volume concentration of $50 \mathrm{vol} . \%$ and $2.5 \mu \mathrm{m}, 10 \mu \mathrm{m}$ and $50 \mu \mathrm{m}$ pigment particle diameter. Grey: Linseed oil; White: Lead white. Sample sizes 4096x100 pixels with a pixel size of $500 \mathrm{~nm}$, corresponding to sample sizes of $2048 \times 50 \mu \mathrm{m}^{2}$. Only a fraction of each sample is shown. Right: Simulated spectra for $3 \mathrm{MeV}$ incident protons at a scattering angle of $165^{\circ}$ for infinitesimally small pigment particles (i.e. atomic mixing of all elements) and pigment particle diameters from $2.5 \mu \mathrm{m}$ to $50 \mu \mathrm{m}$. The edges of $\mathrm{C}, \mathrm{O}$ and $\mathrm{Pb}$ are indicated. 


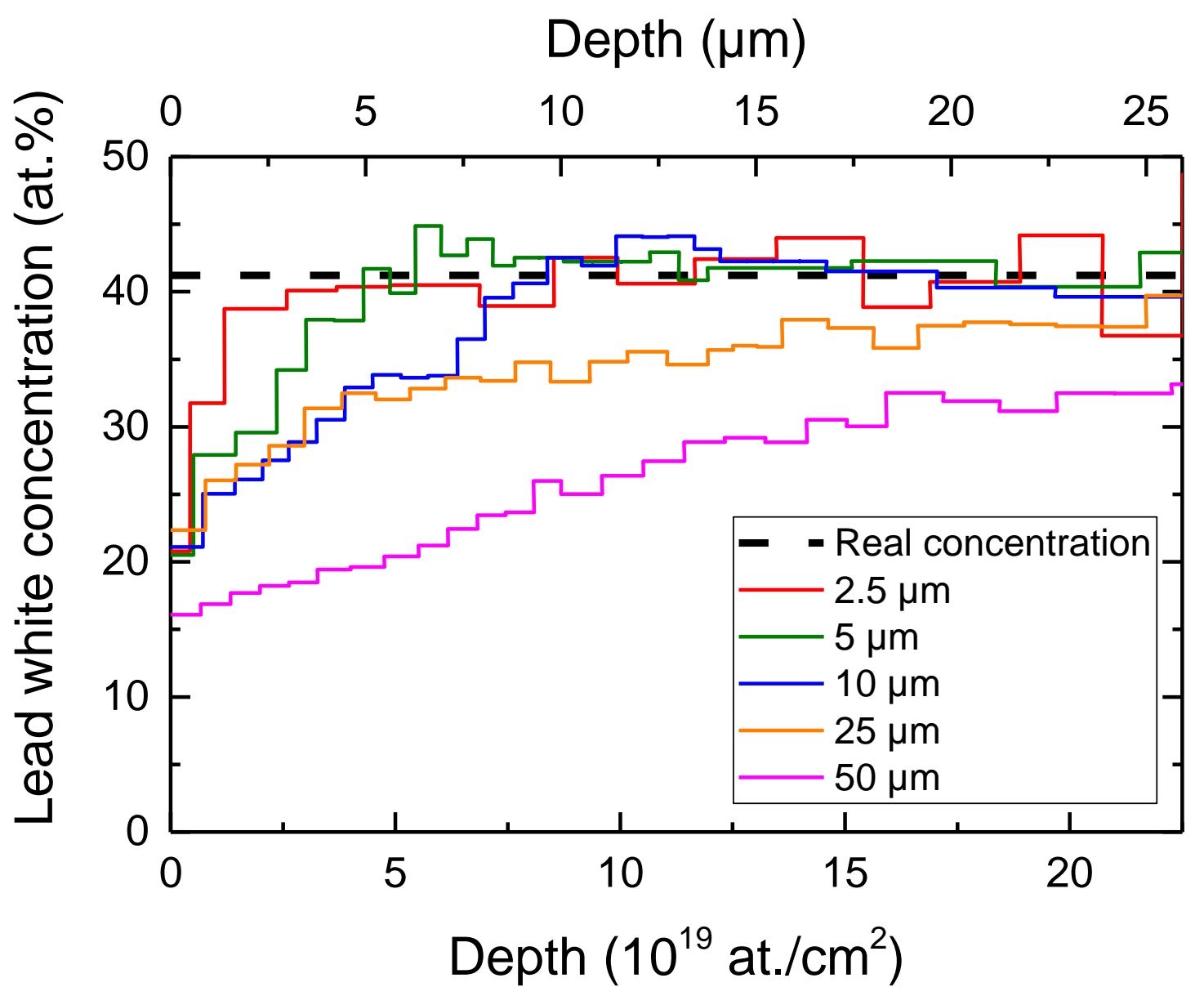

Fig. 6: Depth profiles of lead white in linseed oil derived by fitting a depth profile to the spectra shown in Fig. 5. Dashed line: Real atomic concentration of lead white; Solid lines: Lead white atomic concentrations derived from the spectra for different pigment particle diameters. 

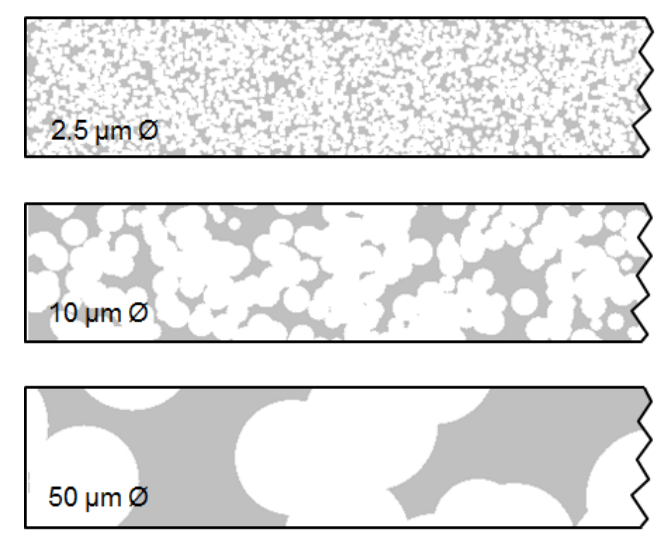

Linseed oil

Lead white

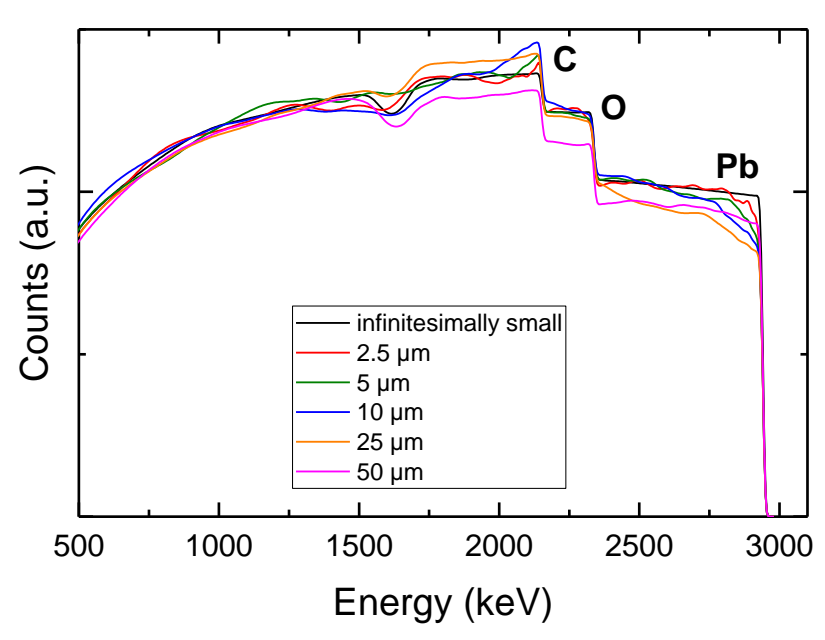

Fig. 7: Left: Typical sample structures for a pigment volume concentration of $70 \mathrm{vol} . \%$ and $2.5 \mu \mathrm{m}, 10 \mu \mathrm{m}$ and $50 \mu \mathrm{m}$ pigment particle diameter. Grey: Linseed oil; White: Lead white. Sample sizes 4096x100 pixels with a pixel size of $500 \mathrm{~nm}$, corresponding to sample sizes of $2048 \times 50 \mu \mathrm{m}^{2}$. Only a fraction of each sample is shown. Right: Simulated spectra for $3 \mathrm{MeV}$ incident protons at a scattering angle of $165^{\circ}$ for infinitesimally small pigment particles (i.e. atomic mixing of all elements) and pigment particle diameters from $2.5 \mu \mathrm{m}$ to $50 \mu \mathrm{m}$. The edges of $\mathrm{C}, \mathrm{O}$ and $\mathrm{Pb}$ are indicated. 
Depth $(\mu \mathrm{m})$

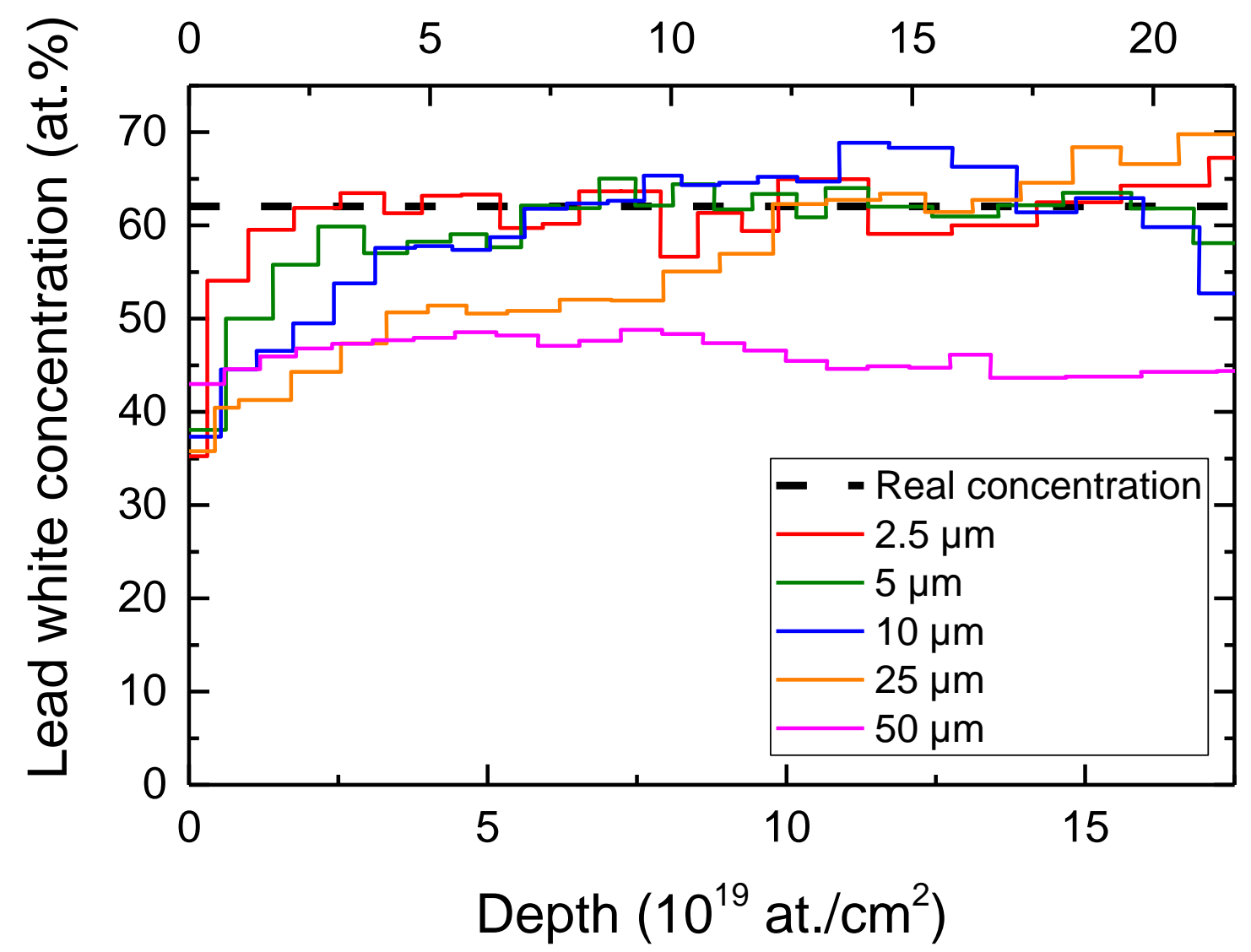

Fig. 8: Depth profiles of lead white in linseed oil derived by fitting a depth profile to the spectra shown in Fig. 7. Dashed line: Real atomic concentration of lead white; Solid lines: Lead white atomic concentrations derived from the spectra for different pigment particle diameters. 

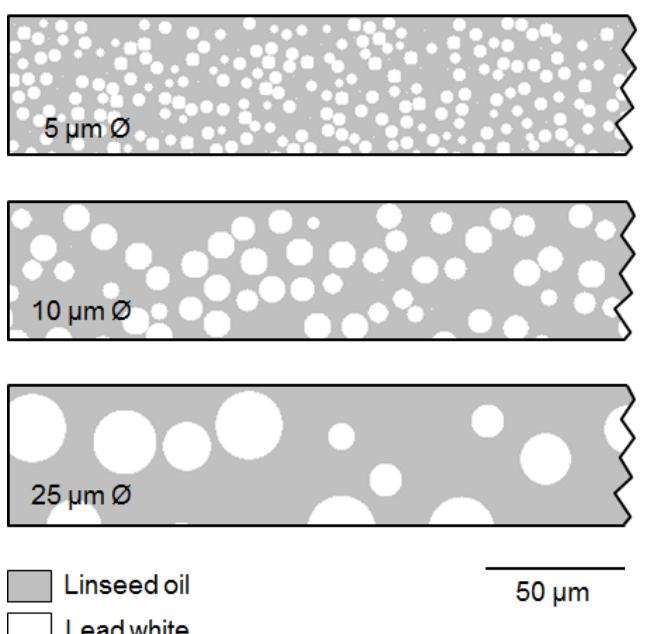

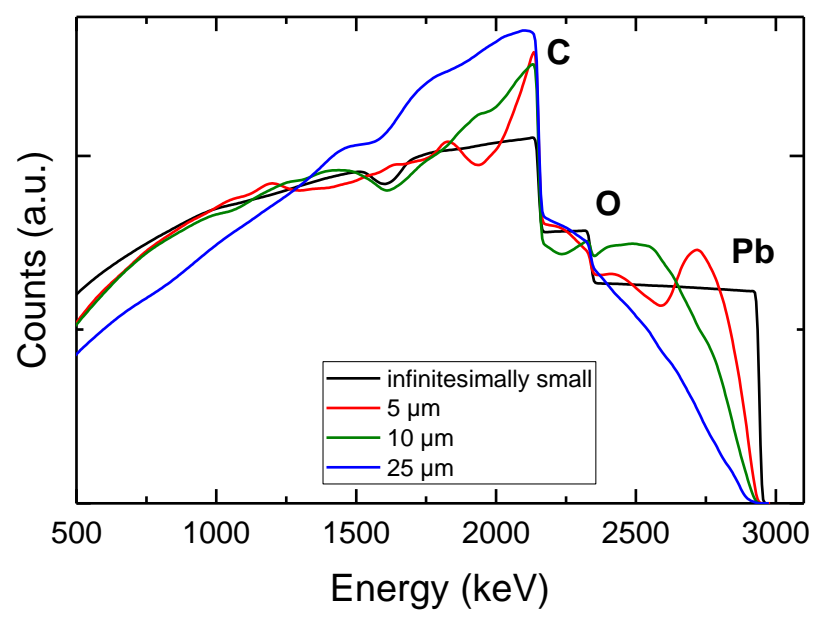

Fig. 9: Left: Typical sample structures for a pigment volume concentration of 30 vol.\%, non-overlapping pigment particles, submersion model with $5 \mu \mathrm{m}, 10 \mu \mathrm{m}$ and $25 \mu \mathrm{m}$ pigment particle diameter. Grey: Linseed oil; White: Lead white. Sample sizes 4096x100 pixels with a pixel size of $500 \mathrm{~nm}$, corresponding to sample sizes of 2048x $50 \mu \mathrm{m}^{2}$. Only a fraction of each sample is shown. Right: Simulated spectra for $3 \mathrm{MeV}$ incident protons at a scattering angle of $165^{\circ}$ for pigment particle diameters of $5 \mu \mathrm{m}, 10 \mu \mathrm{m}$ and $25 \mu \mathrm{m}$. The edges of $\mathrm{C}, \mathrm{O}$ and $\mathrm{Pb}$ are indicated. 


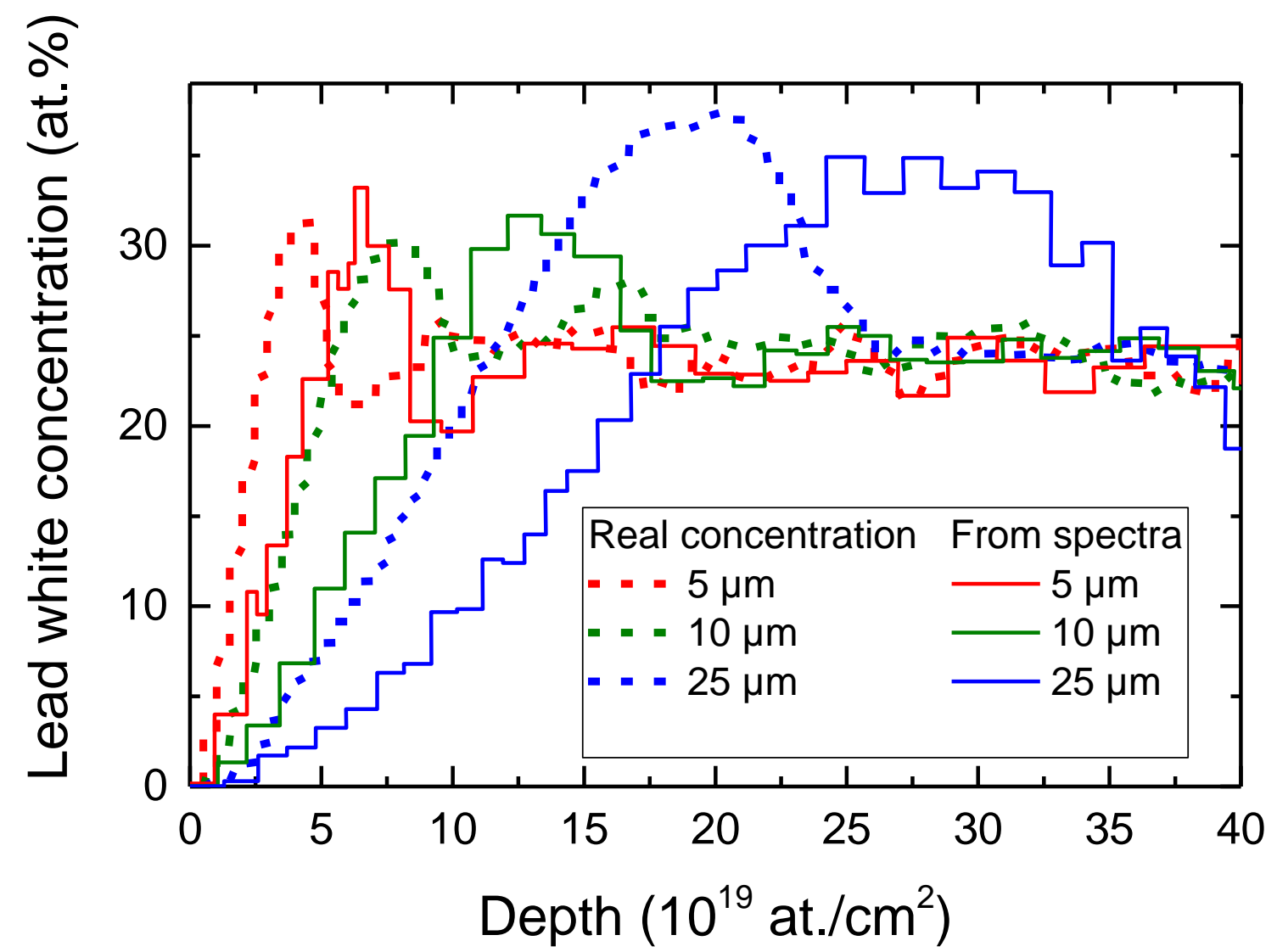

Fig. 10: Depth profiles of lead white in linseed oil derived by fitting a depth profile to the spectra shown in Fig. 9. Dashed lines: Real atomic concentrations of lead white for $5 \mu \mathrm{m}$, $10 \mu \mathrm{m}$ and $25 \mu \mathrm{m}$ pigment particle diameter; Solid lines: Lead white atomic concentrations derived from the spectra. 
Table 1: Pigment atomic and weight fractions for different pigment volume concentrations of lead white in linseed oil. Lead white: $2 \mathrm{PbCO}_{3} \cdot \mathrm{Pb}(\mathrm{OH})_{2}$, mass density $6.14 \mathrm{~g} / \mathrm{cm}^{3}$; Linseed oil: Mean composition $\mathrm{C}_{18} \mathrm{H}_{31} \mathrm{O}_{2}$, mass density $0.93 \mathrm{~g} / \mathrm{cm}^{3}$.

\begin{tabular}{|c|c|c|}
\hline $\begin{array}{c}\text { Pigment volume } \\
\text { concentration } \\
(\%)\end{array}$ & $\begin{array}{c}\text { Pigment atomic } \\
\text { fraction } \\
(\%)\end{array}$ & $\begin{array}{c}\text { Pigment } \\
\text { weight fraction } \\
(\%)\end{array}$ \\
\hline 30 & 23.1 & 73.9 \\
\hline 50 & 41.2 & 86.8 \\
\hline 70 & 62.1 & 93.9 \\
\hline
\end{tabular}

${ }^{1}$ L. Beck, Nucl. Instrum. Methods B 332 (2014) 439

${ }^{2}$ P.A. Mandò, M.E. Fedi, N. Grassi, Eur. Phys. J. Plus 126 (2011) 41

${ }^{3}$ L. Giuntini, Anal. Bioanal. Chem. 401 (2011) 785

${ }^{4}$ L. Beck, C. Jeynes, N.P. Barradas, Nucl. Instrum. Methods B 266 (2008) 1871

${ }^{5}$ L. Beck, L. de Viguerie, Ph. Walter, L. Pichon, P.C. Gutiérrez, J. Salomon, M. Menu, S. Sorieul, Nucl. Instrum. Methods B 268 (2010) 2086

${ }^{6}$ B. Hochleitner, V. Desnica, M. Mantler, M. Schreiner, Spectrochimica Acta Part B 58 (2003) 641

${ }^{7}$ L. de Viguerie, L. Beck, J. Salomon, L. Pichon, Ph. Walter, Anal. Chem. 81 (2009) 7960

${ }^{8}$ E. Pouyet, B. Fayard, M. Salomé, Y. Taniguchi, F. Sette, M. Cotte, Heritage Science (2015) 3:3

${ }^{9}$ J. Salvant, Caractérisation des propriétés physico-chimiques des matériaux de peinture employés par Van Gogh: les peintures blanches, Chimie analytique. Université Pierre et Marie Curie - Paris VI, 2012, https://tel.archives-ouvertes.fr/tel-00834371 (in French)

${ }^{10}$ E. Rauhala, N.P. Barradas, S. Fazinic, M. Mayer, E. Szilágyi, M. Thompson, Nucl. Instrum. Methods B 244 (2006) 436, doi: 10.1016/i.nimb.2005.10.024

${ }^{11}$ M. Mayer, Nucl. Instrum. Methods B 371 (2016) 90, doi: 10.1016/j.nimb.2015.11.032

${ }^{12}$ M. Mayer. SIMNRA user's guide. Tech. Rep. IPP 9/113, Max-Planck-Institut für Plasmaphysik, Garching, 1997

${ }^{13}$ M. Mayer. Nucl. Instrum. Methods B 332 (2014) 176, doi: 10.1016/j.nimb.2014.02.056

${ }^{14}$ J.F. Ziegler, Nucl. Instrum. Methods B 219-220 (2004) 1027

${ }^{15}$ A.F. Gurbich, Nucl. Instrum. Methods B 371 (2016) 27

${ }^{16}$ W. Eckstein, M. Mayer, Nucl. Instrum. Methods B 153 (1999) 337, doi: 10.1016/S0168-583X(98)01011-8

${ }^{17}$ Z. Hajnal, E. Szilágyi, F. Pászti, G. Battistig, Nucl. Instrum. Methods B 118 (1996) 617

${ }^{18}$ F. Schiettekatte, M. Chicoine, Nucl. Instrum. Methods B 371 (2016) 106

${ }^{19}$ P. Malinský, V. Hnatowicz, A. Macková, Nucl. Instrum. Methods B 371 (2016) 101

${ }^{20}$ M. Mayer, P. Malinský, F. Schiettekatte, Z. Zolnai, Nucl. Instrum. Methods B 385 (2016) 65, doi: 10.1016/j.nimb.2016.08.010

${ }^{21}$ T.F. Silva, C.L. Rodrigues, M. Mayer, M.V. Moro, G.F. Trindade, F.R. Aguirre, N. Added, M.A. Rizzutto, M.H. Tabacniks, Nucl. Instrum. Methods B 371 (2016) 86, doi: 10.1016/j.nimb.2015.10.038

${ }^{22} \mathrm{http}: / /$ www.mpi.net/mpitraining/level1/Coatings/Pigments2.asp 